\title{
Median M-Type Radial Basis Function Neural Network
}

\author{
José A. Moreno-Escobar ${ }^{1}$, Francisco J. Gallegos-Funes ${ }^{1}$, \\ and Volodymyr I. Ponomaryov ${ }^{2}$ \\ National Polytechnic Institute of Mexico, \\ Mechanical and Electrical Engineering Higher School \\ ${ }^{1}$ Av. IPN s/n, U.P.A.L.M. SEPI-ESIME, Edif. Z, Acceso 3, Tercer Piso, \\ Col. Lindavista, 07738, Mexico, D. F., Mexico \\ j.augusto.moreno@gmail.com, fgallegosf@ipn.mx \\ ${ }^{2}$ Av. Santa Ana 1000, Col. San Francisco Culhuacan, 04430, Mexico, D. F., Mexico \\ vponomaraipn.mx
}

\begin{abstract}
In this paper we present the capability of the Median M-Type Radial Basis Function (MMRBF) Neural Network in image classification applications. The proposed neural network uses the Median M-type (MM) estimator in the scheme of radial basis function to train the neural network. Other RBF based algorithms were compared with our approach. From simulation results we observe that the MMRBF neural network has better classification capabilities.
\end{abstract}

Keywords: Radial Basis Functions, Rank M-type estimators, Neural Networks.

\section{Introduction}

The artificial neural networks are nonparametric pattern recognition systems that can generalize by learning from examples $[1,2]$. They are particularly useful in problems where decision rules are vague and there is no explicit knowledge about the probability density functions governing sample distributions [1,2].

Recently, we proposed the robust Rank M-type (RM) estimators for image denoising applications [3,4]. The combined RM-estimators use different rank estimators such as the Median, Wilcoxon and Ansari-Bradley-Siegel-Tukey estimators, and the M-estimator with different influence functions to provide better robustness. The performances of the RM-estimators are better in comparison with original R- and M- estimators [3,4].

In this paper is proposed the Median M-Type Radial Basis Function (MMRBF) Neural Network for image classification purposes. The neural network uses the Median M-Type (MM) estimator in the scheme of radial basis function to train the neural network according with the schemes found in the references $[5,6]$.

The rest of this paper is organized as follows. The RBF neural network is presented in section 2. In section 3 we formulate the proposed MMRBF neural network. Experimental results of classification capabilities for simulated images by using our method and other RBF based networks are presented in section 4. Finally, we draw our conclusions in section 5 . 


\section{Radial Basis Function Neural Network}

The Radial Basis Functions (RBF) have been used in several applications for pattern classification and functional modeling [7]. These functions have been found to have very good functional approximation capabilities [7]. The RBF have their fundamentals drawn from probability function estimation theory. In the RBF neural networks each network input is assigned to a vector entry and the outputs correspond either to a set of functions to be modeled by the network or to several associated classes $[1,2,8,9]$. The structure of the RBF neural network is depicted in Figure 1. From Figure 1, each of $N_{k}$ components of the input vector $\mathbf{X}$ feeds forward to $M$ basis functions whose outputs are linearly combined with weights $\left\{\lambda_{j}\right\}_{j=1}^{M}$ into the network output $Y_{k}(\mathbf{X})$.

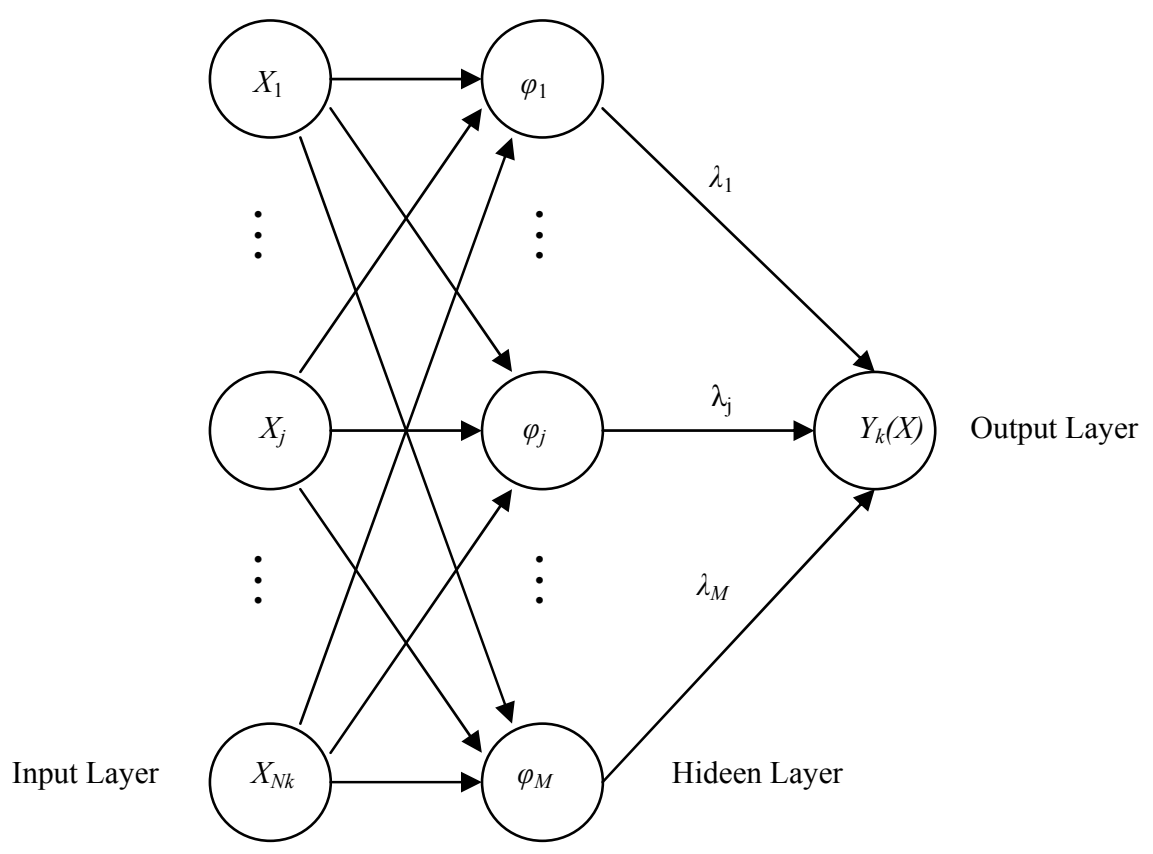

Fig. 1. Structure of Radial Basis Function Neural Network

\subsection{Activation and Output Layer}

Several functions have been tested as activation functions for RBF neural networks. In pattern classification applications the Gaussian function is preferred, and mixtures of these functions have been considered in various scientific fields $[8,9]$.

The Gaussian activation function for RBF neural networks is given by [1,2]:

$$
\phi_{j}(\mathbf{X})=\exp \left[-\left(\mu_{j}-\mathbf{X}\right)^{T} \sum_{j}^{-1}\left(\mu_{j}-\mathbf{X}\right)\right]
$$


where $\mathbf{X}$ is the input feature vector, $\mu_{j}$ is the mean vector and $\Sigma_{j}$ is the covariance matrix of the $j$ th Gaussian function. Geometrically, $\mu_{j}$ represents the center or location and $\Sigma_{j}$ the shape of the basis functions. Statistically, an activation function models a probability density function where $\mu_{j}$ and $\Sigma_{j}$ represent the first and second order statistics. A hidden unit function can be represented as a hyper-ellipsoid in the $\mathrm{N}$ dimensional space.

The output layer implements a weighted sum of hidden-unit outputs [1,2]:

$$
\psi_{k}(\mathbf{X})=\sum_{j=1}^{L} \lambda_{j k} \phi_{j}(\mathbf{X})
$$

where $L$ is the number of hidden units, $M$ is the number of outputs with $k=1, \ldots, M$. The weights $\lambda_{k j}$ show the distribution of the hidden unit $j$ for modeling the output $k$.

\subsection{Learning Techniques of RBF Networks}

Radial Basis Functions have interesting properties which make them attractive in several applications. A combined unsupervised-supervised learning technique has been used in order to estimate the RBF parameters [8]. In the unsupervised stage, $k$ means clustering algorithm is used to find the pdf's parameters, LMS or instead pseudo-inverse matrix can be used in the supervised stage to calculate the weights coefficients in the neural network [8].

\section{Median M-Type Radial Basis Function Neural Network}

In here, we present the use of the RM-estimator as statistic estimation in the Radial Basis Function network architecture. The combined RM-estimators can use different rank estimators such as the Median, Wilcoxon or Ansari-Bradley-Siegel-Tukey [3,4]. In our case we use the MM-estimator with different influence functions.

\subsection{Activation Function}

The Gaussian activation function is the most used function in the RBF networks. We tested with different activation functions and we chose the inverse multiquadratic function $[8,9]$ due this function provided the best results:

$$
\phi_{j}(\mathbf{X})=\frac{1}{\sqrt{\mathbf{X}^{2}+\beta_{j}^{2}}}
$$

where $\mathbf{X}$ is the input feature vector, $\beta_{j}$ is a real constant. In our simulation results $\beta_{j}=1$.

\subsection{K-Means Algorithm}

In our case we use the clustering $k$-means algorithm to estimate the parameters of the MMRBF neural network $[1,2]$. The $k$-means algorithm is used in the unsupervised 
stage. The input feature vector $\mathbf{X}$ is classified in $k$ different clusters. A new vector $\mathbf{x}$ is assigned to the cluster $k$ whose centroid $\mu_{k}$ is the closest one to the vector. The centroid vector is updated according to,

$$
\mu_{k}=\mu_{k}+\frac{1}{N_{k}}\left(\mathbf{x}-\mu_{k}\right)
$$

where $N_{k}$ is the number of vectors already assigned to the $k$-cluster. The centroids can be updated at the end of several iterations or after the test of each new vector. The centroids can be calculated with or without the new vector. By other hand, the steps for the $k$-means algorithm are the following:

\begin{tabular}{cc}
\hline Step 1 & $\begin{array}{c}\text { Select an initial partition with k clusters. Repeat steps 2 through } \\
\text { until the cluster membership stabilizes. }\end{array}$ \\
\hline Step 2 & $\begin{array}{c}\text { Generate a new partition by assigning each pattern to its closest } \\
\text { cluster center. }\end{array}$ \\
\hline Step 3 & Compute new cluster centers as the centroids of the clusters. \\
\hline Step 4 & $\begin{array}{c}\text { Repeat steps } 2 \text { and } 3 \text { until an optimum value of the criterion } \\
\text { function is found. }\end{array}$ \\
\hline
\end{tabular}

\subsection{Median M-Type (MM) Estimator}

The Median M-type (MM) estimator is used in the proposal RBF neural network $[3,4]$. The non-iterative MM-estimator used as robust statistics estimate of a cluster center is given by,

$$
\mu_{k}=\operatorname{med}\{\mathbf{X} \psi(\mathbf{X}-\theta)\}
$$

where $\mathbf{X}$ is the input data sample, $\widetilde{\psi}$ is the normalized influence function $\psi$ : $\psi(\mathbf{X})=\mathbf{X} \tilde{\psi}(\mathbf{X}), \theta=\operatorname{med}\left\{X_{k}\right\}$ is the initial estimate, and $k=1,2, \ldots, N_{k}$.

\subsection{Influence Functions}

In our experiments we use the following influence functions [3]:

- The simple cut (skipped mean) influence function,

$$
\psi_{\text {cut }(r)}(X)=X \cdot 1_{[-r, r]}(X)= \begin{cases}X, & |X| \leq r \\ 0, & \text { otherwise }\end{cases}
$$

- $\quad$ and the Tukey biweight influence function,

$$
\psi_{\mathrm{bi}(r)}(X)= \begin{cases}X^{2}\left(r^{2}-X^{2}\right), & |X| \leq r \\ 0, & \text { otherwise }\end{cases}
$$


where $X$ is a data sample and $r$ is a real constant. The parameter $r$ depends of the data to process and can be change for different influence functions.

\section{Experimental Results}

The described MMRBF neural network has been evaluated, and their performance has been compared with the Simple RBF, $\alpha$-Trimmed Mean RBF, and Median RBF neural networks $[5,6,8]$.

The images used to train the proposed MMRBF neural network and other networks used as comparative are shown in Figure 2. In this figure, the first six images of Group A have common texture or filling which is different form the six first images of Group B. The last two images of each group have a texture or filling that is similar to the opposite group, that is, the last two images of Group A have similar filling than the images of Group B, and vice versa. The main idea here of using textures in figures is to try to simulate medical image textures.

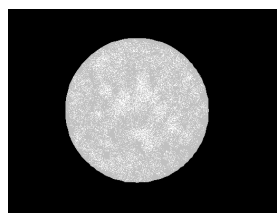

(a)

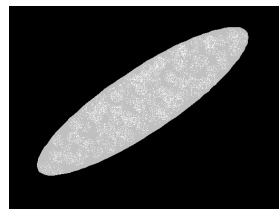

(c)

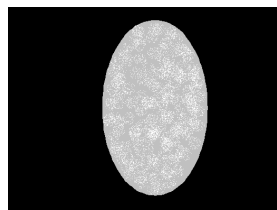

(e)

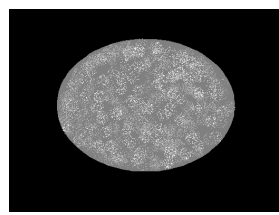

(g)

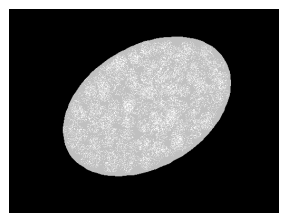

(b)

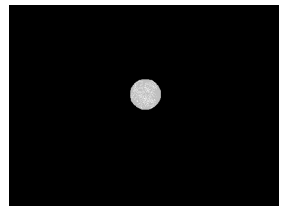

(d)

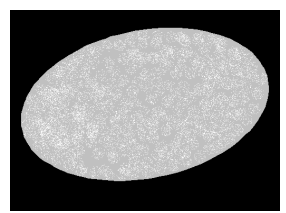

(f)

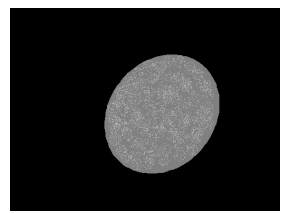

(h)

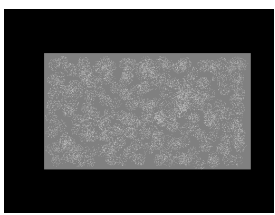

(A)

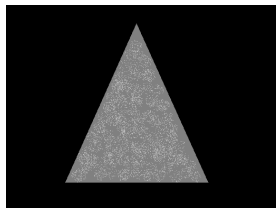

(C)

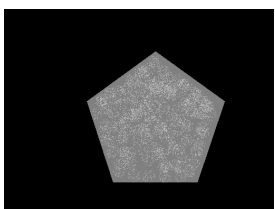

(E)

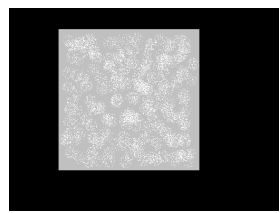

(F)

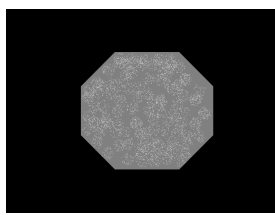

(B)

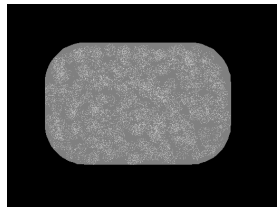

(D)

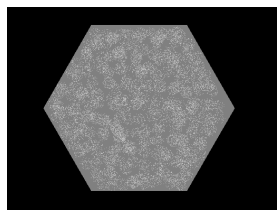

(F)

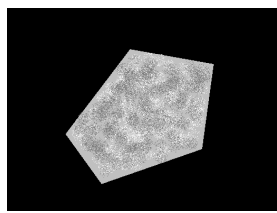

(H)

Fig. 2. Training images 
To train the networks for getting the appropriate pdf's parameters were used 10 images as the ones shown in Figure 2. The objective of this experiment is to classify between 2 main groups:

\begin{tabular}{cc}
\hline Group A & $\begin{array}{c}\text { contains circles and ellipses. The geometric shapes of this group } \\
\text { are denoted with small letters in Figure 2. }\end{array}$ \\
\hline Group B & $\begin{array}{c}\text { contains many kinds of polygons. The geometric shapes of this } \\
\text { group are denoted with capital letters in Figure 2. }\end{array}$ \\
\hline
\end{tabular}

In the segmentation stage were obtained 3 numerical data or characteristics, which are compactness, average gray value, and standard deviation [10,11].

To train the networks implemented in this work we used the block diagram depicted in Figure 3: having the images, the first step is extracting numerical data form them. Afterwards we determined the center of the activation functions. The number of elements used in each activation function depends on the algorithm implemented. The number of elements used to train the comparative Simple RBF, $\alpha-$ Trimmed Mean RBF, and Median RBF neural networks varies in accordance to the training algorithms found in references $[5,6,12]$. In the case of the proposed MMRBF we use eq. (5) in combination with eq. (6) and (7) to determine the elements to be used.

The number of images used to train the networks was 10 (five of each group). The training results are shown in Table 1.

In the test stage we use 30 images (15 of each group), these images are of different form that the images used in the training stage. Figure 4 presents some images used in the test stage. The results obtained are shown in Table 2.

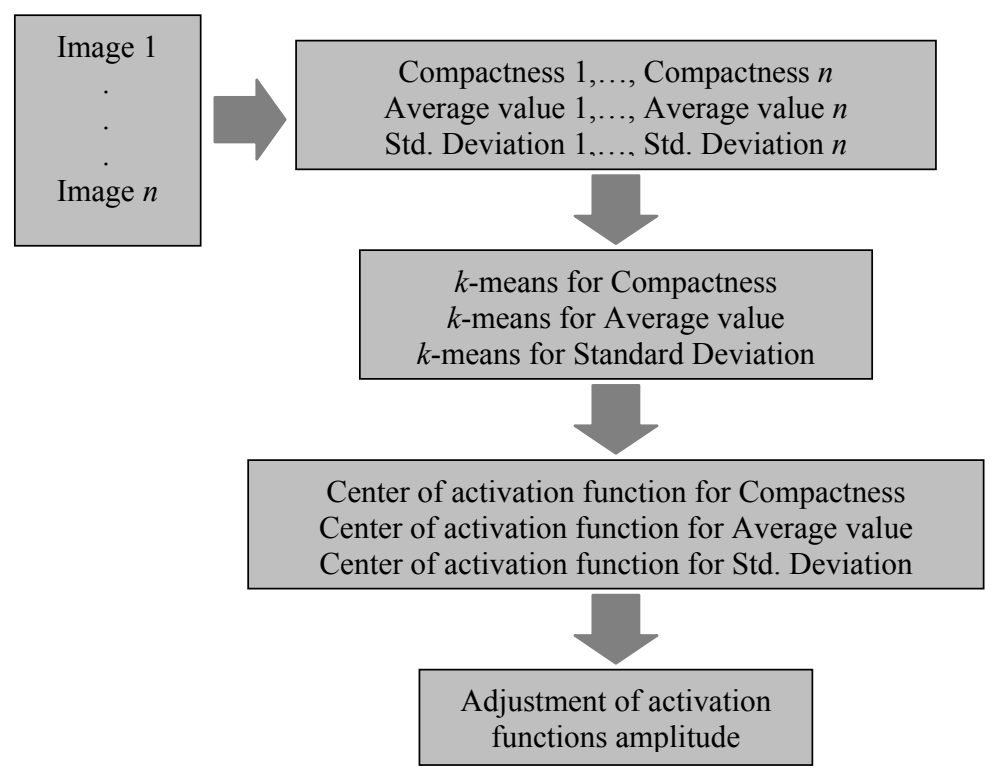

Fig. 3. RBF training block diagram 
Table 1. Results obtained with different RBF algorithms in training stage

\begin{tabular}{cllll}
\hline & & Group A & Group B & Total \\
\hline \multirow{3}{*}{ SIMPLE RBF } & Efficiency & $60 \%$ & $100 \%$ & $80 \%$ \\
\cline { 2 - 5 } & Uncertainty & $0 \%$ & $0 \%$ & $0 \%$ \\
\cline { 2 - 5 } & Error & $40 \%$ & $0 \%$ & $20 \%$ \\
\hline \multirow{3}{*}{ MEDIAN RBF } & Efficiency & $60 \%$ & $100 \%$ & $80 \%$ \\
\cline { 2 - 5 } & Uncertainty & $0 \%$ & $0 \%$ & $0 \%$ \\
\cline { 2 - 5 } & Error & $40 \%$ & $0 \%$ & $20 \%$ \\
\hline \multirow{2}{*}{ M-TRIMMED } & Efficiency & $80 \%$ & $100 \%$ & $90 \%$ \\
\cline { 2 - 5 } & Uncertainty & $0 \%$ & $0 \%$ & $0 \%$ \\
\cline { 2 - 5 } & Error & $20 \%$ & $0 \%$ & $15 \%$ \\
\cline { 2 - 5 } Mimple Cut & Efficiency & $67 \%$ & $100 \%$ & $83.5 \%$ \\
\cline { 2 - 5 } & Uncertainty & $0 \%$ & $0 \%$ & $0 \%$ \\
\cline { 2 - 5 } & Error & $33 \%$ & $0 \%$ & $16.5 \%$ \\
\hline \multirow{2}{*}{ MMRBF } & Efficiency & $67 \%$ & $100 \%$ & $83.5 \%$ \\
\cline { 2 - 5 } Tukey & Uncertainty & $0 \%$ & $0 \%$ & $0 \%$ \\
\cline { 2 - 5 } & Error & $33 \%$ & $0 \%$ & $16.5 \%$ \\
\hline
\end{tabular}

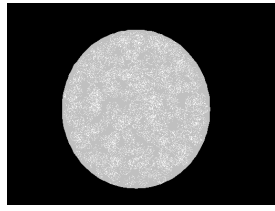

(a)

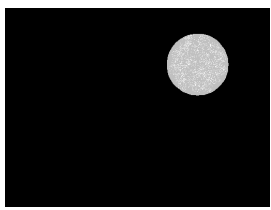

(c)

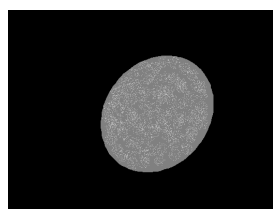

(g)

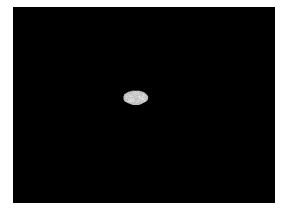

(b)

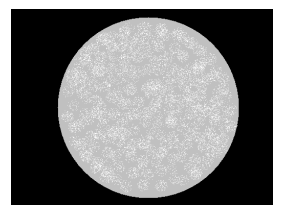

(d)

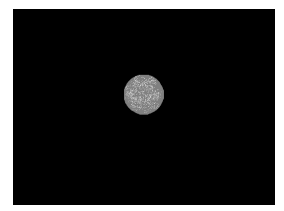

(h)

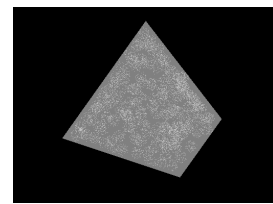

(A)

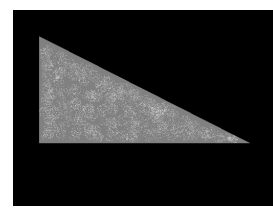

(C)

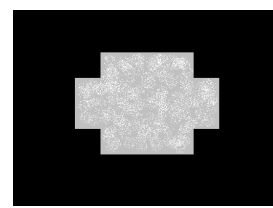

(F)

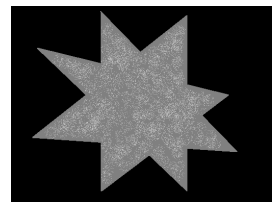

(B)

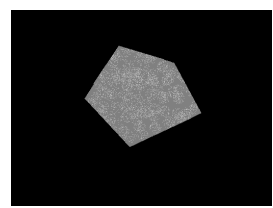

(D)

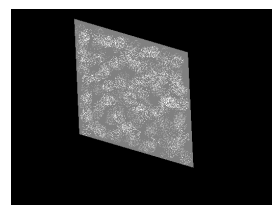

(H)

Fig. 4. Some images used in test stage 
Table 2. Results obtained with different RBF algorithms in test stage

\begin{tabular}{cllll}
\hline & & Group A & Group B & Total \\
\hline \multirow{3}{*}{ SIMPLE RBF } & Efficiency & $67 \%$ & $93 \%$ & $80 \%$ \\
\cline { 2 - 5 } & Uncertainty & $0 \%$ & $0 \%$ & $0 \%$ \\
\cline { 2 - 5 } & Error & $33 \%$ & $7 \%$ & $20 \%$ \\
\hline \multirow{3}{*}{ MEDIAN RBF } & Efficiency & $67 \%$ & $93 \%$ & $80 \%$ \\
\cline { 2 - 5 } & Uncertainty & $0 \%$ & $0 \%$ & $0 \%$ \\
\cline { 2 - 5 } & Error & $33 \%$ & $7 \%$ & $20 \%$ \\
\hline \multirow{2}{*}{$\alpha$-TRIMMED } & Efficiency & $67 \%$ & $87 \%$ & $77 \%$ \\
\cline { 2 - 5 } MEAN RBF & Uncertainty & $0 \%$ & $0 \%$ & $0 \%$ \\
\cline { 2 - 5 } & Error & $33 \%$ & $13 \%$ & $23 \%$ \\
\hline \multirow{2}{*}{ MMRBF } & Efficiency & $67 \%$ & $100 \%$ & $83.5 \%$ \\
\cline { 2 - 5 } & Uncertainty & $0 \%$ & $0 \%$ & $0 \%$ \\
\cline { 2 - 5 } & Error & $33 \%$ & $0 \%$ & $16.5 \%$ \\
\hline \multirow{2}{*}{$\begin{array}{c}\text { MMRBF } \\
\text { Tukey }\end{array}$} & Efficiency & $67 \%$ & $100 \%$ & $83.5 \%$ \\
\cline { 2 - 5 } & Uncertainty & $0 \%$ & $0 \%$ & $0 \%$ \\
\cline { 2 - 5 } & Error & $33 \%$ & $0 \%$ & $16.5 \%$ \\
\hline
\end{tabular}

From previous tables (see Tables 1 and 2) we can appreciate that the difference between algorithms is not big, and that percentages of efficiency, uncertainty and error vary from training stage to test stage. It is evident that if we use more images in the training stage the capabilities of proposed MMRBF neural network can be increased in the test stage in terms of efficiency, uncertainty, and error.

Tables 3 and 4 show a comparison between the RBF algorithms implemented here.

Table 3. Efficiency comparison between the RBF algorithms implemented in training stage

\begin{tabular}{cccc}
\hline Neural Networks & SIMPLE RBF & MEDIAN RBF & $\begin{array}{c}\alpha \text {-TRIMMED } \\
\text { MEAN RBF }\end{array}$ \\
\hline MMRBF Simple Cut & $3.5 \%$ & $3.5 \%$ & $-6.5 \%$ \\
\hline MMRBF Tukey & $3.5 \%$ & $3.5 \%$ & $-6.5 \%$ \\
\hline
\end{tabular}

Table 4. Efficiency comparison between the RBF algorithms implemented in test stage

\begin{tabular}{cccc}
\hline Neural Networks & SIMPLE RBF & MEDIAN RBF & $\begin{array}{c}\alpha \text {-TRIMMED } \\
\text { MEAN RBF }\end{array}$ \\
\hline MMRBF Simple Cut & $3.5 \%$ & $3.5 \%$ & $6.5 \%$ \\
\hline MMRBF Tukey & $3.5 \%$ & $3.5 \%$ & $6.5 \%$ \\
\hline
\end{tabular}

We can see from Tables 3 and 4 that $\alpha$-TRIMMED MEAN RBF had the best efficiency in training stage, but in test stage the best results are given by the proposed MMRBF neural network. 


\section{Conclusions}

We present the MMRBF neural network, it uses the MM-estimator in the scheme of radial basis function to train the proposed neural network. The results obtained with the use of the proposed MMRBF are better than others results obtained with RBF algorithms used as comparative. In future, the proposed network will be extended to real-world datasets such as mammographic imaging.

\section{Acknowledgements}

The authors thank the National Polytechnic Institute of Mexico for its support.

\section{References}

1. Haykin, S.: Neural Networks, a Comprehensive Foundation. Prentice-Hall, Englewood Cliffs (1994)

2. Rojas, R.: Neural Networks: A Systematic Introduction. Springer, Berlin (1996)

3. Gallegos, F., Ponomaryov, V.: Real-time image filtering scheme based on robust estimators in presence of impulsive noise. Real Time Imaging. 8(2), 78-90 (2004)

4. Gallegos-Funes, F., Ponomaryov, V., De-La Rosa, J., ABST,: M-type K-nearest neighbor (ABSTM-KNN) for image denoising. IEICE Trans. Fundam. Electron. Commun. Comput. Sci. E88-A(3), 798-799 (2005)

5. Bors, A.G., Pitas, I.: Median radial basis function neural network. IEEE Trans. Neural Networks. 7(6), 1351-1364 (1996)

6. Bors, A.G., Pitas, I.: Object classification in 3-D images using alpha-trimmed mean radial basis function network. IEEE Trans. Image Process. 8(12), 1744-1756 (1999)

7. Buhmann, M.D.: Radial Basis Functions: Theory and Implementations. Cambridge Monographs on Applied and Computational Mathematics (2003)

8. Karayiannis, N.B., Weiqun Mi, G.: Growing radial basis neural networks: merging supervised and unsupervised learning with network growth techniques. IEEE Trans. Neural Networks. 8(6), 1492-1506 (1997)

9. Karayiannis, N.B., Randolph-Gips, M.M.: On the construction and training of reformulated radial basis function neural networks. IEEE Trans. Neural Networks. 14(4), $835-846$ (2003)

10. Ritter, G.: Handbook of Computer Vision Algorithms in Image Algebra. CRC Press, Boca Raton-New York (2001)

11. Myler, H.R., Weeks, A.R.: The Pocket Handbook of Image Processing Algorithms in C. Prentice-Hall, Englewood Cliffs (1993)

12. Musavi, M.T., Ahmed, W., Chan, K.H., Faris, K.B., Hummels, D.M.: On the training of radial basis function classifiers. Neural Networks. 5, 595-603 (1992) 